\author{
I. Ciepał - B. Kłos - St. Kistryn - E. Stephan - A. Biegun · K. Bodek · A. Deltuva \\ E. Epelbaum - M. Eslami-Kalantari - A. C. Fonseca - J. Golak - V. Jha - N. Kalantar-Nayestanaki \\ H. Kamada • G. Khatri · Da. Kirillov • Di. Kirillov • St. Kliczewski - A. Kozela • M. Kravcikova \\ H. Machner - A. Magiera - G. Martinska - J. Messchendorp • A. Nogga • W. Parol \\ A. Ramazani-Moghaddam-Arani - B. J. Roy - H. Sakai - K. Sekiguchi • I. Sitnik · R. Siudak \\ R. Skibiński · R. Sworst · J. Urban · H. Witała · J. Zejma
}

\title{
Investigation of the Three-Nucleon System Dynamics in the Deuteron-Proton Breakup Reaction
}

Received: 29 October 2013 / Accepted: 1 February 2014 / Published online: 19 February 2014

(C) The Author(s) 2014. This article is published with open access at Springerlink.com

\begin{abstract}
Precise and large sets of cross section, vector $A_{x}, A_{y}$ and tensor $A_{x x}, A_{x y}, A_{y y}$ analyzing power data for the ${ }^{1} H(\mathbf{d}, p p) n$ breakup reactions were measured at 100 and $130 \mathrm{MeV}$ deuteron beam energies with the SALAD and BINA detectors at KVI and the Germanium Wall setup at FZ-Jülich. Results are compared with various theoretical approaches which model the three-nucleon system dynamics. The cross section data reveal a sizable three-nucleon force (3NF) and Coulomb force influence. In case of the analyzing powers very
\end{abstract}

This work was supported by the Foundation for Polish Science-MPD program, co-financed by the European Union within the European Regional Development Fund, Małopolskie Centrum Przedsiebiorczości-Project "Doctus-Małopolski fundusz stypendialny dla doktorantòw", the Polish 2013-2015 science founds as research Project No. 2012/05/E/ST2/02313 and funding from Jagiellonian University within SET project - the project is co-financed by the European Union.

I. Ciepał $(\bowtie) \cdot$ K. Bodek · J. Golak · G. Khatri · St. Kistryn · A. Magiera · W. Parol · R. Skibiński · R. Sworst · H. Witała · J. Zejma

Institute of Physics Jagiellonian University, 30059 Kraków, Poland

E-mail: izabela.ciepal@uj.edu.pl

B. Kłos · E. Stephan

University of Silesia, 40007 Katowice, Poland

A. Biegun · N. Kalantar-Nayestanaki · J. Messchendorp

Kernfysisch Versneller Instituut, 9747 AA Groningen, The Netherlands

St. Kliczewski · A. Kozela $\cdot$ R. Siudak

Institute of Nuclear Physics 31342 Kraków, Poland

M. Eslami-Kalantari

Faculty of Physics, Yazd University, Yazd, Iran

A. Ramazani-Moghaddam-Arani

Faculty of Physics, University of Kashan, Kashan, Iran

Da. Kirillov · H. Machner · A. Nogga

Forschungszentrum Jülich, Institut für Kernphysik, 52425 Jülich, Germany

E. Epelbaum

Institut für Theoretische Physik II, Ruhr-Universität Bochum, 44780 Bochum, Germany

A. Deltuva - A. C. Fonseca

Centro de Física Nuclear da Universidade de Lisboa, 1649-003 Lisbon, Portugal 
low sensitivity to these effects was found and the data are well describe by $2 \mathrm{~N}$ models only. For $A_{x y}$ at 130 $\mathrm{MeV}$, serious disagreements were observed when 3NF models are included in the calculations.

\title{
1 Introduction
}

One of the key issues of modern nuclear physics is the investigation of the forces acting between nucleons. Properties of three-nucleon $(3 \mathrm{~N})$ systems at medium energies are determined by the pairwise nucleonnucleon (NN) interaction, which is a dominant component. The interaction models are created based on the meson exchange theory or phenomenology. These so-called realistic NN potentials, like CD Bonn, Nijmegen or Argonne AV18 are able to predict observables for $2 \mathrm{~N}$ systems with very high precision. To test thoroughly these models, systems with more than just two nucleons are needed. The simplest is the $3 \mathrm{~N}$ system, which can be used to test dynamics and features of the $3 \mathrm{~N}$ Hamiltonian. Such systems can be studied in details with the use of the deuteron breakup reaction, offering a very unique laboratory in which even very subtle dynamical effects like the $3 \mathrm{NF}$, Coulomb force or relativistic components can be studied.

The theoretical predictions of observables are nowadays obtained via exact solutions of the $3 \mathrm{~N}$ Faddeev equations for the given interaction model, like for example realistic NN potentials supplemented with $3 \mathrm{NF}$ models (TM99 3NF [1] or Urbana IX 3NF [2]), or the 3N system dynamics is treated within the coupledchannels (CC) approach [3] with a single $\Delta$-isobar degree of freedom (d.o.f.), which generates also certain $3 \mathrm{NF}$ effects. Another alternative way comes from chiral perturbation theory (ChPT) where the nuclear potential is obtained by a systematic expansion in terms of momenta, and the many-body interactions appear naturally at increasing order. The non-vanishing $3 \mathrm{NF}$ enters at the next-to-next-to-leading (NNLO) order $[4,5]$, which is numerically fully developed. Within the CC formalism the Coulomb interaction was implemented into the calculations for the first time [6]. Recently, a consistent theoretical treatment of a phenomenological $3 \mathrm{NF}$ and the Coulomb force has been achieved also for the AV18+UIX potential [7] that allows to investigate the role of both effects to a high level of accuracy. Moreover, the relativistic treatment of the breakup reaction in the $3 \mathrm{~N}$ system was developed for calculations using the NN potential [8] and this approach has been also extended to calculations including the 3NF [9]. To verify the model predictions, the deuteron breakup reaction can be used as a valuable experimental tool. The final state of the reaction is complex enough to test different ingredients of few-nucleon system dynamics, which enter with varying strength in certain phase-space regions. The extensive and precise data sets which were obtained in a series of new generation experiments are used to distinguish between different interaction contributions and to test the corresponding predictions.

\author{
H. Kamada \\ Department of Physics, Kyushu Institute of Technology, Kitakyushu 804-8550, Japan \\ V. Jha $\cdot$ B. J. Roy \\ BARC, Bombay 400 085, India \\ Da. Kirillov · Di. Kirillov · I. Sitnik \\ JINR, Dubna 141980, Russia \\ M. Kravcikova \\ Technical University, 04101 Kosice, Slovakia \\ G. Martinska · J. Urban \\ P. J. Safarik University, 04154 Kosice, Slovakia \\ B. J. Roy \\ Universität Bonn, 53113 Bonn, Germany \\ H. Sakai \\ University of Tokyo, Bunkyo, Tokyo 1130033, Japan \\ K. Sekiguchi \\ Tohoku University, Sendai 9808578, Japan
}




\section{Experimental Setups}

The ${ }^{1} H(\mathbf{d}, p p) n$ breakup reactions were studied in different phase-space regions with the use of three detection systems: the SALAD [10] and BINA [11] at KVI and the Germanium Wall (GeWall) [12] at FZ-Jülich. Results of the analyzing powers were obtained with the BINA and SALAD detectors at polarized deuteron beam energies of 100 [13] and $130 \mathrm{MeV}$ [14], respectively. The cross-section data [15,16,18-20] were measured using the SALAD and GeWall detectors at a deuteron beam energy of $130 \mathrm{MeV}$. The BINA detector offered access to almost the full phase-space, whereas the SALAD and GeWall setups covered only forward polar angles.

The SALAD detector consisted of a three-plane multi-wire proportional chamber (MWPC) and two layers of a segmented scintillator hodoscope: horizontal $\Delta E$ and vertical stopping $E$ detectors. The acceptance of the setup covered the region of polar angles from $12^{\circ}$ to $40^{\circ}$ and the full range of azimuthal angles. The liquid hydrogen target was placed inside the scattering chamber. The BINA apparatus was constructed as an upgraded version of SALAD and possessed two main parts called Wall and Ball. The Wall inherited most parts and features from SALAD, covering the same angular range and built of the same MWPC and modified $\Delta E$ and $E$ hodoscopes. The backward part is ball-shaped and consists of 149 phoswich detectors which cover polar angles between $40^{\circ}$ and $160^{\circ}$. The Ball plays two roles: particle detector and scattering chamber. In the measurements a liquid target $\left(\mathrm{LH}_{2}\right)$ was used.

The GeWall setup at the Research Center in Jülich (FZJ) consisted of three high-purity semiconductor position sensitive germanium detectors. Two different types of the detectors were used: a thin transmission detector "Quirl" with excellent spatial resolution to determine the position and energy loss ( $\Delta E$ detector) of the passing charged particles, and two thick energy detectors E1 and E2 with excellent energy resolution. The angular acceptance of the apparatus was $5^{\circ}-14^{\circ}$ for the polar and $2 \pi$ for the azimuthal angles.

\section{Results}

In order to search for subtle dynamical effects in few-body systems a precise and systematic database is necessary, making measurements very demanding. Our new-generation experiments fulfilled these conditions and provided a very rich set of differential cross-section, vector $A_{x}, A_{y}$ and tensor $A_{x x}, A_{x y}, A_{y y}$ analyzing power data for the breakup reaction. The cross-section data were obtained for about 80 [15-17] and 145 [1820] geometries, defined by the polar angles of the two outgoing protons, $\theta_{1}, \theta_{2}$, and their relative azimuthal angle $\varphi_{12}$ for an energy of $65 \mathrm{MeV} /$ nucleon. The vector and tensor analyzing power data were measured in 90 geometries for each of the tensor and vector analyzing powers [14] with the SALAD detector. Moreover, measurements with BINA and GeWall delivered additional sets of data for vector analyzing powers at deuteron beam energies of $100 \mathrm{MeV}$ [13] and $130 \mathrm{MeV}$ [12], respectively.

The cross sections obtained for $65 \mathrm{MeV} /$ nucleon at KVI were compared with theoretical predictions and revealed both a significant influence of $3 \mathrm{NF}[15,17]$ and Coulomb effects [16]. The data $[15,17]$ confirmed the importance the $3 \mathrm{NF}$ for understanding of the $3 \mathrm{~N}$ system dynamics. Inclusion of this additional force in the calculations leads generally to a better description of the cross-section data.

The other important and crucial piece of the dynamics turned out to be the electromagnetic interaction in some parts of the phase space. Only calculations which take into account both dynamical components, i.e., the $3 \mathrm{NF}$ and the Coulomb force, are able to remove disagreements between data and calculations. Adding the electromagnetic force into the calculations does not essentially change the quality of the data description at large relative energy $\left(E_{r e l}\right)$ of the two breakup protons. However at small $E_{r e l}$ values the observed discrepancies are almost totaly removed. The inclusion of $3 \mathrm{NF}$ and Coulomb effects into the calculations does not remove completely the disagreement between data and theoretical predictions. There are still some discrepancies at large $E_{r e l}$ which can be interpreted as indication that parts of the dynamics are missing, either relativistic effects or unresolved problems in our understanding of the $3 \mathrm{NF}$ structure.

The Coulomb force effects were studied in more details in a dedicated experiment at FZJ [18-20], focusing on a very narrow part of the phase space. This study was extremely important due to the fact that the electromagnetic interaction reveals itself quite strongly in this region. The results confirmed the importance of the Coulomb force and showed that only models containing the missing part of the dynamics reproduce the data in a proper way, see Fig. 1. To trace the influence of the electromagnetic processes globally, the dependence of $\chi^{2}$ per d.o.f. was studied as a function of $E_{\text {rel }}$, see Fig. 2. The $\chi^{2}$ was calculated as the squared difference between the experimental cross section value and the theoretical one and divided by the uncertainty of the 

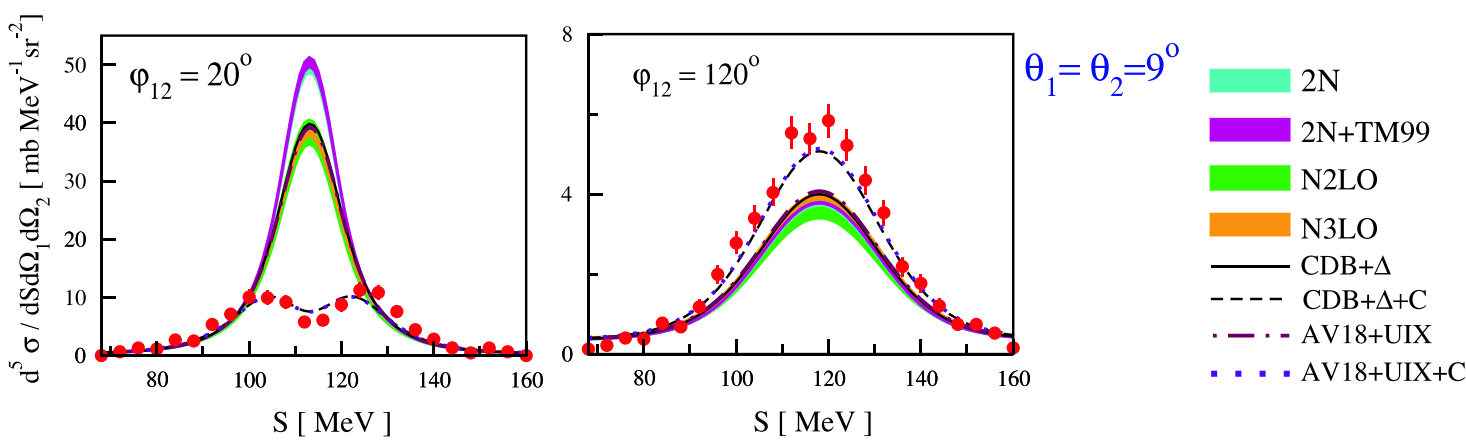

Fig. 1 Differential cross-section data for the $d-p$ breakup reaction at $130 \mathrm{MeV}$ at two different kinematical configurations (specified in the panels) in which significant Coulomb force effects are observed. The data are compared to various theoretical calculations, described in the legend

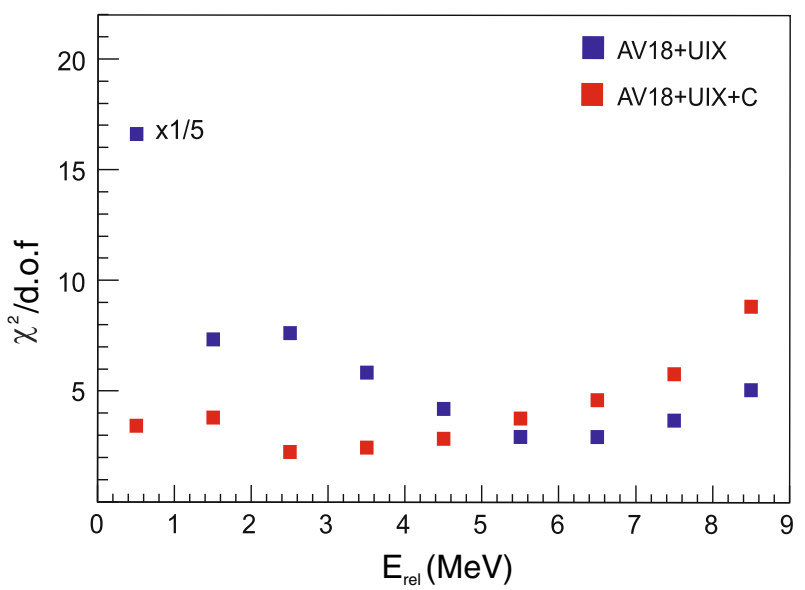

Fig. 2 Quality of description of the cross-section data with the calculations based on the AV18 NN potential combined with the Urbana IX 3NF (blue squares) and, in addition, with the Coulomb force included (red squares). The dependence of $\chi^{2} /$ d.o.f on the kinetic energy of the relative motion of the two breakup protons is shown. The point with a very large value of $\chi^{2} /$ d.o.f is scaled down by a factor of $1 / 5$, as indicated in the panel (color figure online)

experimental datum. In the case of very small $E_{r e l}$ the Coulomb effects are extremaly high. For very large $E_{\text {rel }}>5.5 \mathrm{MeV}$ the discrepancies are still present, but become much smaller. The results obtained revealed a quite strong influence of Coulomb force effects on the breakup reaction. It has been proven that only the predictions containing the Coulomb component are able to reproduce the data in a correct way. Figure 3 presents the seize of the predicted relative Coulomb effect on the cross-section data (relative difference of the results obtained with $\left(\sigma_{t h+e f f}\right)$ and without $\left(\sigma_{t h}\right)$ the Coulomb force) as a function of $E_{r e l}$. The effect is at the level of up to about $40 \%$.

The measurements provided a very rich set of data for the vector $A_{x}, A_{y}$ and tensor $A_{x x}, A_{x y}, A_{y y}$ analyzing powers as well. In this case the dynamics of the system was also investigated by use of the $\chi^{2}$ and $E_{\text {rel }}$ variables. For the $A_{x}$ and $A_{y}$ analyzing powers the $\chi^{2} /$ d.o.f. was analyzed as a function of $E_{\text {rel }}$ (see Fig. 4). The results obtained at $65[14]$ and $50 \mathrm{MeV} /$ nucleon [13] are well reproduced by $2 \mathrm{~N}$ calculations in the whole phase-space studied. This observation implies that the observables are practically insensitive to details of the nuclear dynamics. In case of the tensor analyzing powers [14] certain discrepancies are observed at $130 \mathrm{MeV}$. The theory predicts significant effects of the TM99 3NF. However there are configurations in which inclusion of the TM99 3NF leads to a worse agreement with the experimental data. Such discrepancies were found especially for the $A_{x y}$ tensor analyzing power. This suggests that important ingredients are missing in the spin part of the $3 \mathrm{NF}$ model.

For the data [12] measured with the GeWall detector, the values obtained for $A_{x}$ and $A_{y}$ are very small and they do not reveal any interesting effects connected with the $3 \mathrm{~N}$ dynamics. In general, the data confirm the theoretical calculations, though they can be described by the calculations limited to the pairwise NN interaction only. 


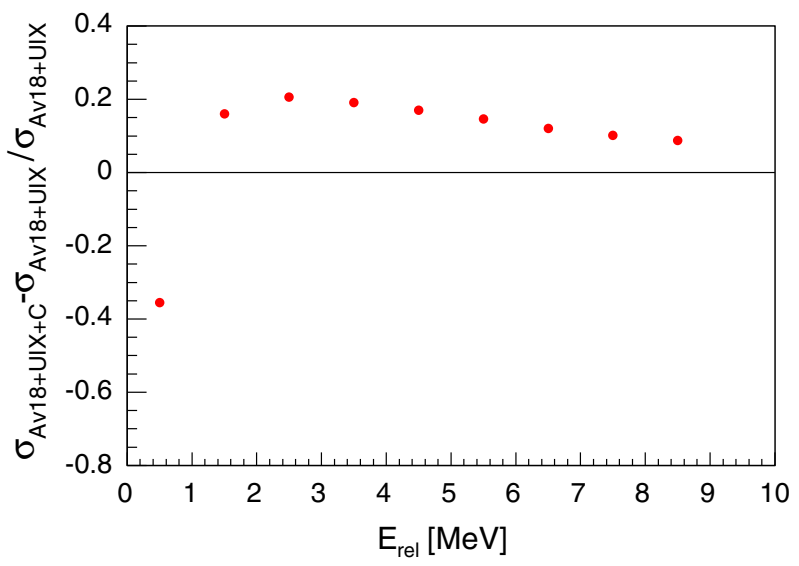

Fig. 3 Net effect of the Coulomb force on the theoretical breakup cross section at $65 \mathrm{MeV} /$ nucleon presented as function of the $E_{\text {rel }}$ variable. The calculations are matched to the acceptance of the GeWall detector

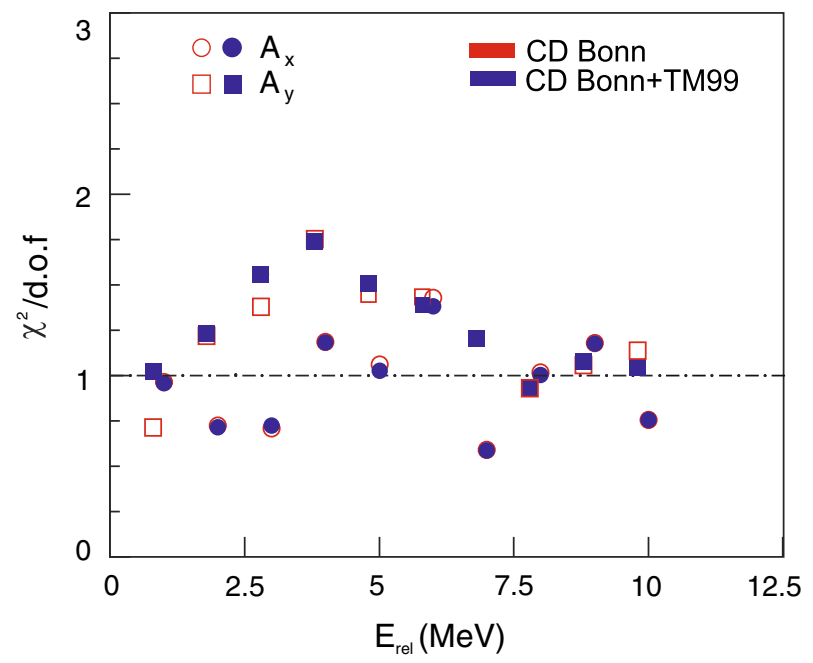

Fig. 4 Quality of description of the vector analyzing powers of the deuteron-proton breakup reaction at $50 \mathrm{MeV} / \mathrm{nucleon}$ given by various models (as indicated in the panel), presented as dependence of $\chi^{2} /$ d.o.f on kinetic energy of the relative motion of the two breakup protons $\left(E_{r e l}\right)$

\section{Summary and Outlook}

Precise and systematic studies of the breakup reaction in a large part of the phase space are very important for understanding of the interaction between nucleons in few-nucleon systems. Theoretical approaches, which try to model the interaction, need a very precise and large experimental database. The currently available database has to be verified and extended. Using the theoretical predictions different pieces of the dynamics can be studied separately and also their mutual interplay can be investigated. The data were obtained at several beam energies, and in general, they confirm the modern calculations. However there are still some problems with our understanding of the current models of the $3 \mathrm{NF}$. Moreover, there is a strong need to have complete theoretical treatments, including all ingredients of the $3 \mathrm{~N}$ system dynamics (3NF, Coulomb interaction, relativistic effects).

Recent years brought significant progress in the theory. The relativistic treatment of the breakup reaction in $3 \mathrm{~N}$ systems was developed using $\mathrm{NN}$ potential in [8] and this approach has also been extended to calculations with $3 \mathrm{NF}$ effects in [9]. At higher energies only very scarce data for the breakup observables exist. The data are randomly distributed over the phase space and are not sufficient to draw any global conclusions concerning the influence of relativity or $3 \mathrm{NF}$ effects. To test the theoretical achievements an experiment was performed with the WASA detector at FZ-Jülich at deuteron beam energies of 340, 380 and $400 \mathrm{MeV}$. The results will enable the study of the evolution of relativistic and $3 \mathrm{NF}$ effects on the cross section at higher energies. This 
will put strong constraints onto the theoretical calculations and will allow to improve the quality of the existing few-nucleon potential models.

New experiments to study the $3 \mathrm{~N}$ system dynamics are planned, including also investigations of the threebody system in the four-body environment. A new scientific program concentrated on the investigation of the few-nucleon systems dynamics at not too high energies was proposed to be carried out with the use of the BINA detector at the Cyclotron Center Bronowice in Cracow, Poland. This scientific center offers the possibility to continue the experimental program of investigation of the few nucleon systems dynamics with the use of proton beams in the energy range between 70 and $230 \mathrm{MeV}$.

Open Access This article is distributed under the terms of the Creative Commons Attribution License which permits any use, distribution, and reproduction in any medium, provided the original author(s) and the source are credited.

\section{References}

1. Coon, S.A., Han, H.K.: Reworking the Tucson-Melbourne three-nucleon potential. Few Body Syst 30, 131 (2001)

2. Pudliner, B.S., et al.: Quantum Monte Carlo calculations of nuclei with A < 7. Phys. Rev. C 56, 1720 (1997)

3. Deltuva, A., Machleidt, R., Sauer, P.U.: Realistic two-baryon potential coupling two-nucleon and nucleon- $\Delta$-isobar states: fit and applications to three-nucleon system. Phys. Rev. C 68, 024005 (2003)

4. Entem, D.R., Machleidt, R.: Accurate nucleon-nucleon potential based upon chiral perturbation theory. Phys. Lett. B 524, 93-98 (2002)

5. Epelbaum, E.: Few-nucleon forces and systems in chiral effective field theory. Prog. Part. Nucl. Phys. 57, 645-741 (2006)

6. Deltuva, A., Fonseca, A.C., Sauer, P.U.: New calculations schemes for proton-deuteron scattering including the Coulomb interaction. Phys. Rev. C 73, 057001 (2006)

7. Deltuva, A.: Momentum-space calculation of proton-deuteron scattering including Coulomb and irreducible three-nucleon forces. Phys. Rev. C 80, 064002 (2009)

8. Skibiński, R., Witała, H., Golak, J.: Relativistic effects in exclusive neutron-deuteron breakup. Eur. Phys. J. A 30, 369 (2006)

9. Witała, H., et al.: Three-nucleon force in relativistic three-nucleon Faddeev calculations. Phys. Rev. C 83, 044001 (2011)

10. Stephan, E., et al.: Precise set of tensor analyzing power $T_{20}$ data for the deuteron-proton breakup at $130 \mathrm{MeV}$. Eur. Phys. J. A 42, 13 (2009)

11. Ramazani-Moghaddam-Arani, et al.: Elastic proton-deuteron scattering at intermediate energies. Phys. Rev. C 78, 014006 (2008)

12. Ciepał, I., et al.: Vector analyzing powers of deuteron-proton elastic scattering and breakup at $130 \mathrm{MeV}$. Phys. Rev. C 85, 017001 (2012)

13. Stephan, E. et al.: Vector analyzing powers of the deuteron-proton elastic scattering and breakup at $100 \mathrm{MeV}$. Eur. Phys. J. A 49, 36 (2013)

14. Stephan, E., et al.: Vector and tensor analyzing powers in deuteron-proton breakup at $130 \mathrm{MeV}$. Phys. Rev. C 82, 014003

15. Kistryn, St., et al.: Systematic study of three-nucleon force effects in the cross section of the deuteron-proton breakup at $130 \mathrm{MeV}$. Phys. Rev. C 72, 044006 (2005)

16. Kistryn, St., et al.: Evidence of the Coulomb-force effects in the cross sections of the deuteron-proton breakup at $130 \mathrm{MeV}$. Phys. Lett. B 641, 23-27 (2006)

17. Kalantar-Nayestanaki, N., et al.: Signatures of three-nucleon interactions in few-nucleon systems. Rep. Prog. Phys. 75, 016301

18. Ciepał, I., et al.: Studies of the three-nucleon system dynamics in the deuteron-proton breakup reaction. EPJ Web Conf. 37, 09011 (2012)

19. Ciepał, I., et al.: Investigations of few-nucleon system dynamics in medium energy domain. Few Body Syst. 601, 1-5 (2013)

20. Kłos, B., et al.: Systematic studies of the three-nucleon system dynamics in the deuteron-proton breakup reaction. Acta Phys. Pol. B 44, 345 (2013) 